\title{
Free Form Surface Five-axis 3D Printing
}

\author{
WEI Shengli ${ }^{1, a}, \quad$ CAO Ling ${ }^{2, b}, \quad$ LI Yuan ${ }^{3, c}$, \\ ${ }^{1,2,3}$ School of Computer Science \& Information Engineering, \\ Anyang Institute of Technology, Anyang, Henan Province, China

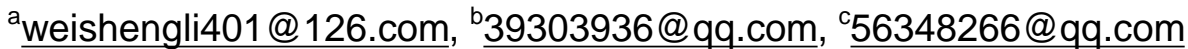

\section{Keywords: 3D Printing; Free Form Surface; Five-axis; NURBS}

Abstract: Being directed against the shortage of existing 3D printing, a five-axis 3D printer is designed, and a free form surface five-axis 3D printing method is presented. In this method, a five-axis 3D printer is used, a curved surface instead of a flat surface is used as the initial surface, each layer being printed is a curved surface rather than a plane. Three path planning methodologies are introduced according to different shape curved surface, contour line methodology, vertical cross section methodology and fixed parameter methodology. Then translational coordinates and rotating coordinates of the five-axis printer are calculated in term of some interpolation algorithm. The Non-uniform Rational B Spline (NURBS) technology is mainly used as the curved surface description method, and the fixed parameter path planning methodology is taken as an example to discuss the approach and algorithm of the free from surface five-axis printing method. The approach and algorithm include surface reverse engineering, path planning and coordinate computing etc.. The free form surface five-axis 3D printing method can avoid the step effects which always take place in existing $3 \mathrm{D}$ printing. The method is effective for the printing in which the bottom of the workpiece being printed is a sphericity or other curved surface shape or in which the main purpose is to cover a or many printing material layer on the underprop's surface. This method also can be used to spray some material on a curved surface.

\section{Preface}

The manufacturing way by Numerical Control(NC) is usually called material reducing manufacturing, because NC usually works by means of cutting, milling or punching and so on. It is clear that this way will cause waste of material. The 3D printing technology is so-called additive material manufacturing, which works with stacking layer by layer[1,2]. This method does not cause material waste. Compared with $\mathrm{NC}$ technology, 3D printing is a new manufacturing method[3]. Because of its special advantages, 3D printing will have a place in the manufacturing field in the future. It can replace existing manufacturing methods in some fields, even is called the fourth technological revolution[4].

At present, the existing 3D printing works by spraying and solidifying material layer by layer on a horizontal plane to form a final product. The 3D printer working in this way usually has a simple structure and has been widely used. But in some cases, this structure does not fully meet the requirements. Firstly, "Step Effects" is one of the important errors in existing 3D printing which cause the printing precision is not high. Secondly, if the bottom of the final product or workpiece being printed is a spherical cavity or other shapes such as a curved surface instead of a plane, the existing 3D printer usually with a horizontal bottom plate which is called baseplate does not work. In this case, a 3D printer with a curved surface support rather than a horizontal bottom plate will be a good choice. So far the researchs on 3D printing or rapid prototyping mainly focus on the 
hierarchical intersection algorithm[5-11], and the research on this aspect still relatively lacks. Based on the above requirements, a Free Form Surface Five-axis 3D Printing Method is proposed. In this method, the top surface of the base support is a curved surface. First, the surface equation shall be obtained, usually by reverse engineering. Then in terms of the surface equation, the walking path of the printer head is computed. After that, some interpolation algorithms are used to calculate the coordinates of the moving printer head. The control system and the motors of the 3D printer can drive the printer head to move and spray according to the coordinate values. This method is applicable to the printing of the final product of which the bottom is not a plane but a curved surface.

\section{General Description}

The mechanism of a 3D printer is the same as that of a NC system. It is necessary to calculate the moving coordinates of each step of the print head by the way of interpolation calculation. In the existing 3D printing technology, slicing operation on a 3D model shall be done ahead. In the slicing operation, intersection computation is needed, and each slicing layer data is stored. Path planning and interpolation computing are conducted in each slicing layer and at last G-code is generated. The existing 3D printer uses G-code to carry out the job. That is, the existing 3D printer is driven by a digital 3D model. In the proposed free form surface 3D printing system, the five-axis 3D printer works by the top surface of the base support instead of a digital 3D model. The five-axis 3D printer head coordinates are carried out by the surface initially. The top surface of the base support is called the Initial Basis Surface here. Based on this surface, the first layer is printed. The first layer also become a surface by which the second printing layer can be done. For the initial basis surface, the first layer surface is called the printing surface, and for the second layer, it is called the basis surface. And the like, all layers can be done. The number of the layers depends on the design thickness of the final product and the thickness of each layer. When in printing, it is usually required that the printer head should be perpendicular to the tangent plane of the printing surface, so the axis of the printer head should coincide with the normal vector of the printing point, as shown in Fig. 1 . The printing points are on the printing surface not the basis surface. Because the printing layer has a certain thickness, it actually is the equidistant surface of the basis surface. In order to ensure that the axis of the printer head coincides with the normal vector, five axis linkage is needed. Besides three translational coordinates $\mathrm{x}, \mathrm{y}$ and $\mathrm{z}$, two rotating coordinates $\mathrm{A}$ and $\mathrm{C}$ are needed. The structure of the five-axis 3D printer is shown in Fig. 2.

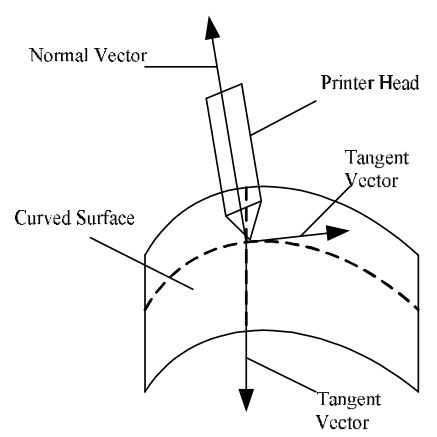

Fig.1 Free Form Surface Printing

The five-axis 3D printer is composed of base, bracket, guide rail, bottom plate and printer head. The printer head can move along the direction of the $\mathrm{x}$ axis and the $\mathrm{y}$ axis along the guide rail, and 
the bottom plate can move along the $\mathrm{z}$ axis. The printer head can also be rotated in the direction of the $\mathrm{A}$ and $\mathrm{C}$ axes. The five axes $\mathrm{x}, \mathrm{y}, \mathrm{z}, \mathrm{A}$, and $\mathrm{C}$ can be controlled with linkage.

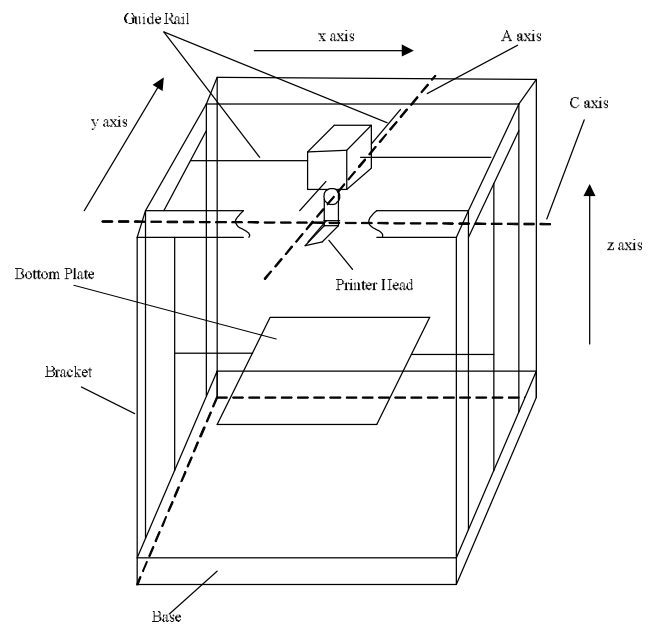

Fig.2 Five-axis Printer

Most of the existing 3D printers print on a flat surface and each layer is a flat. The five-axis 3D printing method is different from the conventional printing method of horizontal slicing. In this method, a support is needed to place on the bottom plate of the five-axis printer. The top of the support is a curved surface which usually forms the bottom shape of the final product being printed. The five-axis printer works on this surface, so each layer printed will be a curved surface which is like the top surface of the support. So the top surface of the support is called Initial Basis Surface. At some time, the surface of each printed layer is almost like the offset surface of the initial basis surface. The surface can be described in any way. Here it is represented in NURBS method. The working process of the five-axis 3D printer is as follows:

Step 1: To obtain the surface equation of the initial basis surface. Sometimes the surface equation is known. In this case, subsequent calculation can be carried out in terms of this surface equation. But in most cases, the surface equation of the initial basis surface is unknown. Under this condition, scattered points cloud data of the initial basis surface can be extracted by scanning the surface. With these scattered points cloud data, reverse engineering technology is used to reconstruct the initial basis surface equation. Here the initial basis surface equation is represented with NURBS technology.

Step 2: Planning printing path. After getting the initial basis surface equation in step 1, there are three methodologies as following to be chosen to plan the printing path.

(1) Contouring method. If cutting the initial basis surface with a group of horizontal planes, a set of contour lines formed by the initial basis surface intersecting with the group of horizontal planes will be gotten. The contour lines can be as the printing path.

(2) Vertical section method. A set of curves is formed by a set of vertical planes cutting the initial basis surface. and the curves can be used as the printing path.

(3) Fixed parameter method. For a double parametric surface, if a parameter is set as a constant, the surface equation is changed to a curve equation decided by another parameter. For example, for a surface represented by NURBS surface equation, if one of the two parameters is set as a constant, it will change to a NURBS curve equation decided by another parameter. By this way, if a parameter is dispersed into a series of fixed values, and a set of NURBS curves is finally obtained. These curves can be used as the printing path. 
Step 3: Computing point coordinates. After the path is planned, the parameters of the curve are dispersed into a series of values along each path. Point coordinates on each path are computed out in terms o some interpolation algorithms.

Step 4: Work out printer head translational coordinates. Because each printing layer has a certain thickness, the point coordinates on the surface computed out in step 3 are different from the printer head translational coordinates. The print head translational coordinates can be calculated according to point coordinates, printing layer thickness and the normal vector at the point.

Step 5: Calculating rotating coordinates. In the above steps, three translational coordinates of the printer head are worked out. For the five-axis 3D printer, besides the three coordinates of $x, y$ and $\mathrm{z}$, two rotating axes are needed. According to the rotation transformation matrix, the coordinates of the two rotating axes are obtained. After the five coordinates are calculated out, the five-axis 3D printer can implement printing operation in terms of these coordinates.

\section{NURBS Surface Definition}

A NURBS surface is usually defined as Eq. 1[12].

$$
C(u, \omega)=\frac{\sum_{i=0}^{n} \sum_{j=0}^{m} B_{i, k}(u) B_{j, l}(\omega) W_{i, j} V_{i, j}}{\sum_{i=0}^{n} \sum_{j=0}^{m} B_{i, k}(u) B_{j, l}(\omega) W_{i, j}}
$$

Here $\mathbf{V}_{i, j}$ is the Control Point Vector, $W_{i, j}$ is the weight factor, $B_{i, k}(u)$ and $B_{j, l}(\omega)$ are the base function of the B Spline. The node vector $\mathbf{U}$ with $u$ direction and the node vector $\mathbf{N}$ with $\omega$ direction are usually as follows:

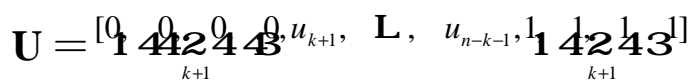

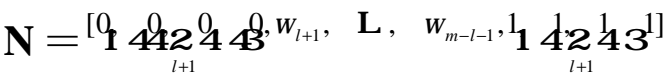

Each printing layer can be defined by NURBS method, but for conventional surfaces, such as a sphere or a hyperboloid, the conventional quadric surface definition method can also be adopted.

\section{Inversing Surface Equation}

The free form surface five-axis 3D printing method needs to get the initial basis surface equation firstly. That is, it needs the top surface equation of the support. Sometimes the surface equation is known, in this case, subsequent calculation can be carried out in terms of this surface equation. But in most cases, the surface equation of the initial basis surface is unknown. Under this condition, scattered points cloud data of the initial basis surface can be extracted by scanning the surface. With these scattered points cloud data, reverse engineering technology is used to reconstruct the initial basis surface equation. There are many literatures research on the inverse surface equation based on point cloud [13]. Here, double NURBS curve method is adopted in the reverse engineering. For a NURBS curve, its equation is as follows [12].

$$
C(u)=\frac{\sum_{i=0}^{n} B_{i, k}(u) W_{i} \mathbf{V}_{i}}{\sum_{i=0}^{n} B_{i, k}(u) W_{i}}
$$


Here, $B_{i, k}(u)$ is the B Spline base function, $\mathbf{V}_{i}$ is the control vector, and $W_{i}$ is the weight factor. Eq. 2 can be rewritten as Eq. 3 and 4.

$$
\begin{aligned}
& C(u)=\sum_{i=0}^{n} \mathbf{V}_{i} R_{i, k}(u) \\
& R_{i, k}=\frac{W_{i} B_{i, k}(u)}{\sum_{j=0}^{n} W_{j} B_{j, k}(u)}
\end{aligned}
$$

The NURBS curve interpolation is to work out all points on the curve $\mathrm{C}$ according to control vector $\mathbf{V}_{i}$. Whereas, the reverse engineering is to find control vector $\mathbf{v}_{i}$ according to some points that the curve $\mathrm{C}$ must pass through which are usually referred to as type value point. After smoothing the point cloud data, some points are selected as the type value points. The Eq. 3 can be changed to Eq. 5 .

$$
\sum_{i=0}^{n} \mathbf{V}_{i} R_{i, k}(u)=C_{i}
$$

According to Eq. 5, the control vector $\mathbf{v}_{i}$ can be found. Nevertheless, in order to solve Eq. 5, some boundary conditions need to be supplemented. The boundary conditions include the tangent condition, the free end condition and the closed curve condition. In general, even if with the boundary conditions, the solution of Eq. 5 is still difficult. Here a NURBS equation is simplified as a quasi uniform B spline to solve the problem. For the quasi uniform B spline, the inverse equation is written as the form of the following matrix[12]:

$$
\left[\begin{array}{cccccccc}
b_{1} & c_{1} & & & & & & \\
1 / 4 & 7 / 12 & 1 / 6 & & & & & \\
& 1 / 6 & 2 / 3 & 1 / 6 & & & & \\
& & \cdots & \cdots & \cdots & & & \\
& & & \cdots & \cdots & \cdots & & \\
& & & & 1 / 6 & 2 / 3 & 1 / 6 & \\
& & & & & 1 / 6 & 7 / 12 & 1 / 4 \\
& & & & & & a_{n} & b_{n}
\end{array}\right]\left[\begin{array}{c}
\mathbf{V}_{1} \\
\mathbf{v}_{2} \\
\mathbf{v}_{3} \\
\cdots \\
\mathbf{V}_{n-1} \\
\mathbf{V}_{n} \\
\mathbf{V}_{n+1}
\end{array}\right]=\left[\begin{array}{c}
d_{1} \\
C_{1} \\
C_{2} \\
\cdots \\
C_{n-2} \\
C_{n-1} \\
d_{n}
\end{array}\right]
$$

Here $\mathbf{V}_{0}=C_{0}, \mathbf{V}_{n+1}=C_{n} . b_{1}, c_{1}, a_{n}, b_{n}, d_{1}$ and $d_{n}$ take different values in term of different boundary conditions. In tangent condition, the values will be as follows.

$$
\begin{array}{lll}
b_{1}=3, & c_{1}=0, & d_{1}=C_{1}^{\prime}+3 C_{1} \\
a_{n}=0, & b_{n}=3, & d_{n}=3 C_{n}-C_{n}^{\prime}
\end{array}
$$

In free end condition, the values will be as follows.

$$
\begin{aligned}
& b_{1}=9, \quad c_{1}=-3, \quad d_{1}=6 C_{1} \\
& a_{n}=-3, \quad b_{n}=9, \quad d_{n}=6 C_{n}
\end{aligned}
$$

Eq. 6 is a triangular matrix, it can be solved by chasing method.

According to the above method, control vector is obtained. After this step, the weight factors can be set to 1 initially, and then the control vector and weight factor can be adjusted slightly until they meet the requirements and to make the curve or surface more smooth and more suitable for the design. 


\section{Computing printer head coordinates}

Computing translational coordinates In case a initial basis surface is defined as follows.

$$
C(u, \omega)=\frac{\sum_{i=0}^{n} \sum_{j=0}^{m} B_{i, k}(u) B_{j, l}(\omega) W_{i, j} V_{i, j}}{\sum_{i=0}^{n} \sum_{j=0}^{m} B_{i, k}(u) B_{j, l}(\omega) W_{i, j}}
$$

The range of change of the $u$ direction node vector $\mathbf{U}$ and $\omega$ direction node vector $\mathbf{N}$ are all from 0 to 1 .

(1) Let the parameter $\omega$ be 0 , then the above Eq. 7 changes to a NURBS curve equation with the parameter $u$, and it is shown as Eq. 8 .

$$
C(u, 0)=\frac{\sum_{i=0}^{n} B_{i, k}(u) \mathbf{D}_{i} W_{i}}{\sum_{i=0}^{n} B_{i, k}(u) W_{i}}
$$

Here

$$
\mathbf{D}_{i}=\frac{\sum_{j=0}^{m} B_{j, l}(\omega) W_{i, j} \mathbf{V}_{i, j}}{\sum_{j=0}^{m} B_{j, l}(\omega) W_{i, j}}
$$

The value of $\omega$ in Eq. 9 is 0 .

(2) Let the parameter $u$ be 0 , then the value of the point $C(0,0)$ on the surface can be computed according to Eq. 8 and 9. There is a printing layer different between this point and its corresponding printer head point in the normal vector direction. The normal vector can be calculated by Eq. 10 as follows.

$$
\gamma=\frac{\boldsymbol{\alpha} \times \boldsymbol{\beta}}{|\boldsymbol{\alpha} \times \boldsymbol{\beta}|}
$$

Here, $\gamma$ is the normal vector, $\boldsymbol{\alpha}$ and $\boldsymbol{\beta}$ are two tangent vector of the two parameter direction. If the printer head is perpendicular to the tangent plane of the printed surface, the printer head coordinates can be determined by the equation below.

$$
P=C(0,0)+d \bullet \gamma
$$

Here $P$ is the printer head coordinate vector, $C(0,0)$ is the point value vector and $d$ is the printing layer thickness.

(3) Let parameter $u$ add an increment $\Delta u$, and this increment can be determined by a variety of methods, such as equal spacing method, equal step length method, error constraint method, etc.. Replace the new value of the parameter $u$ into the Eq. 8 and 9, the next point coordinates can be obtained and the position of the next printer head can be calculated. And the like, until the parameter $u$ is 1 .

(4) Let parameter $\omega$ add an increment $\Delta \omega$, and this increment can be determined by a variety of methods, such as equal spacing method, error constraint method, etc.. And like this, another NURBS curve equation is gotten.

$$
C(u, 0+\Delta \omega)=\frac{\sum_{i=0}^{n} B_{i, k}(u) \mathbf{D}_{i} W_{i}}{\sum_{i=0}^{n} B_{i, k}(u) W_{i}}
$$




$$
\mathbf{D}_{i}=\frac{\sum_{j=0}^{m} B_{j, l}(\omega) W_{i, j} \mathbf{V}_{i, j}}{\sum_{j=0}^{m} B_{j, l}(\omega) W_{i, j}}
$$

In the above equation the value of parameter $\omega$ is $\Delta \omega$ 。

(5) Then let parameter $u$ be 1 , the point with parameter $u$ and $\omega$ being $(1, \Delta \omega)$ can be worked out. The starting point of printer head on the new curve can be calculated by equation $P=C+d \bullet \gamma$.

(6) Let parameter $u$ has a reduction $\Delta u$, and this reduction can be determined by a variety of methods, such as equal spacing method, equal step length method, error constraint method, etc.. Replace the new value of the parameter $u$ into the Eq. 8 and 9, the next point coordinates can be obtained and the position of the next printer head can be calculated by the equation $P=C+d \bullet \gamma$. And the like, until the parameter $u$ is 0 .

(7) Repeat the above process, and the whole surface can be printed out.

(8) Using the printed surface which is finished in above seven process as the next basis surface, repeat the above process to complete the printing of second layer.

(9) Until all layers are printed.

Computing rotating coordinates For the five-axis 3D printer, besides the three position or translational coordinates of $\mathrm{x}, \mathrm{y}$ and $\mathrm{z}$, two rotating axes are needed. The two rotating axes are $\mathrm{A}$ axis and $\mathrm{C}$ axis, and they can be calculated with rotation transformation matrix. Let the rotation motion of $\mathrm{A}$ axis is $A(u, \omega)$ and $\mathrm{C}$ axis is $C(u, \omega)$, the rotation translation matrix of $\mathrm{A}$ axis and $\mathrm{C}$ axis are $M(A(u, \omega))$ and $M(C(u, \omega))$, then[14]

$$
\begin{aligned}
& \mathbf{M}(A(u, \omega))=\left[\begin{array}{ccc}
1 & 0 & 0 \\
0 & \cos (A(u, \omega)) & -\sin (A(u, \omega)) \\
0 & \sin (A(u, \omega)) & \cos (A(u, \omega))
\end{array}\right] \\
& \mathbf{M}(C(u, \omega))=\left[\begin{array}{ccc}
\cos (C(u, \omega)) & -\sin (C(u, \omega)) & 0 \\
\sin (C(u, \omega)) & \cos (C(u, \omega)) & 0 \\
0 & 0 & 1
\end{array}\right]
\end{aligned}
$$

Let the initial values of the normal vector, $\mathrm{A}$ axis and $\mathrm{C}$ axis are $(0,0,1), 0$ and 0 , then the axis vector of the printer head $\mathbf{i}$ can be:

$$
\mathbf{i}=\left(i^{x}, i^{y}, i^{z}\right)^{T}=\mathbf{M}(C(u, \omega)) \mathbf{M}(A(u, \omega))(0,0,1)^{T}
$$

The rotation motion $A(u, \omega)$ and $C(u, \omega)$ can be calculated by

$$
\begin{aligned}
& A(u, \omega)=\arccos \left(i^{z}\right) \\
& C(u, \omega)=\arctan \left(-\frac{i^{x}}{i^{y}}\right)
\end{aligned}
$$

In the condition that the printer head is always perpendicular to the printing surface, the axis vector of the printer head $\mathbf{i}$ can be replaced with the normal vector $\gamma$ which is used in Eq. 10 and 11.

\section{Conclusion}

The application of 3D printing is becoming more and more extensive, and 3D printers suitable for different needs are constantly emerging. 3D printers with different structure and features are 
suitable for different application scenarios. Aiming at the shortcomings of the existing 3D printing methods which are basically flat printing, a five-axis 3D printer is designed, and a free form surface five-axis printing method is proposed. The method adopts a five-axis printer, and uses free-form surface instead of plane as the basic printing surface. According to the different characteristics of the initial basis surface, three path planning methods are adopted, and the translational coordinates and rotating coordinates of the five axes are computed. Generally, NURBS is used as the free form surface description method and the fixed parameter methodology is selected as the path planning method to discuss the calculating steps and algorithms of free form surface five-axis 3D printing process. The free form surface five-axis 3D printer proposed in this paper is suitable for the case that the printing surface is not a plane but a curved surface. This printing method can avoid the "step effects" which is a common problem in existing 3D printing method and can affect the accuracy of the printed workpieces or products. For a workpiece being printed of which the bottom is a curved surface instead of a plane, this free form surface five-axis 3D printing method will be more effective. For the application that some material needs to be spayed to a curved surface, this method can also be qualified, so this method can be extended to applications of spraying a coating on a curved surface too.

\section{Acknowledgements}

This work was financially supported by Henan Province Science and Technology Key Projects (162102210130).

\section{References}

[1] ZHAO Chen. Design and implementation of the slicing software of 3D printer[D]. Nanjing: Southeast University, 2009.

[2] ZHOU Manyuan. A non-uniform self-adaption slicing method[J]. Computer Engineering and Applications, 2005,3:72-74.

[3] DU Peng, WANG Bin. Analysis of the development trend of 3D printers[J]. China Science and Technology Information, 2014.7:141-143.

[4] LIU Juyan. 3D printer is changing manufacturing rules[J]. World Science, 2012.7:22-24.

[5] ZHAO Jibin, LIU Weijun. Recent progress in slicing algorithm of rapid prototyping technology[J]. Computer Integrated Manufacturing Systems, 2009,2:209-219.

[6] MA Jinhua, LU Zhangping. The realization of direct-adaptive slicing approach based on AutoCAD[J]. Machinery Design \& Manufacture, 2007, 7: 100-103.

[7] YANG Guang, LIU Weijun, WANG Wei, etc.. Research on the rapid slicing algorithm based on STL topology construction[J]. Modern Manufacturing Engineering, 2009,10:32-35.

[8] WANG Chunxiang, LI Zhenhua. The Optimization and Application of the STL Model Layer Algorithm[J]. Machinery Design \& Manufacture, 2013,3:87-90.

[9] HE Qaing, ZHANG Shusheng, BAI Xiaoliang. Fast repairing errors in STL files[J]. Application Research of Computers, 2009,5:1983-1984.

[10] LI Zhanli, LIANG Dong, LI Dichen, etc.. Algorithm for Rapid Slicing Based on the Information Inheriting[J]. Journal of Xi'an Jiaotong University, 2002,1:43-46.

[11] WANG Chunxiang, HAO Zhibo. The Uniform Thickness Hierarchical Algorithm of Rapid Prototyping Technology STL Model[J].Machinery Design \& Manufacture, 2014,4:133-136.

[12] ZHU Xinxiong. Free form curve and surface modeling technology[M]. Beijing: Science Press, 2000.

[13] LIU Hong, FAN Ruyuan, ZENG Liang etc.. NURBS freeform surface construction from points-cloud of sectional feature[J]. Modern Manufacturing Engineering, 2012,5:81-84.

[14] ZHOU Ji, ZHOU Yanhong. NC Machining Technology[M]. Beijing: National Defense Industry Press, 2002. 